\title{
Procedures for Computing One- and Two-Dimensional Integrals of Functions With Rapid Irregular Oscillations
}

\author{
By David Levin
}

\begin{abstract}
A collocation procedure for efficient integration of rapidly oscillatory functions is presented. The integration problem is transformed into a certain O.D.E. problem, and this is solved by a collocation technique. The method is also extended to two-dimensional integration, and some numerical results are appended showing the efficiency of the method in handling difficult cases of rapid irregular oscillations.
\end{abstract}

1. The Procedure for One-Dimensional Integrals. We consider integrals of the form

$$
I=\int_{a}^{b} f(x) e^{i q(x)} d x
$$

where $f$ is smooth and "nonoscillatory" and $\left|q^{\prime}(x)\right| \gg(b-a)^{-1}$. Two practical methods for evaluating rapidly oscillatory integrals are described in [1], the use of approximation as in Filon's method [3], [4] and the speedup method of Longman [5]. Formally both methods are applicable to any integral of the form (1.1), but their best performance is for the case of a constant frequency $q^{\prime}=W$. In this note we present an efficient method which is applicable for cases of varying frequency $q^{\prime}$ using only a small number of values of $f$ and $q^{\prime}$ in $[a, b]$ and the values $q(a)$ and $q(b)$.

The proposed method follows the spirit of Filon's method. It is based upon the fact that if $f$ were of the form

$$
f(x)=i q^{\prime}(x) p(x)+p^{\prime}(x) \equiv L^{(1)} p(x), \quad a \leqslant x \leqslant b,
$$

then the integral could be evaluated directly as

$$
\begin{aligned}
I & =\int_{a}^{b}\left(i q^{\prime}(x) p(x)+p^{\prime}(x)\right) e^{i q(x)} d x=\int_{a}^{b} \frac{d}{d x}\left(p(x) e^{i q(x)}\right) d x \\
& =p(b) e^{i q(b)}-p(a) e^{i q(a)} .
\end{aligned}
$$

Equation (1.2) can be considered as a differential equation for $p(x)$, and any solution of this equation can be used in (1.3) for evaluating $I$. The general solution of this equation is

$$
p(x)=e^{-i q(x)}\left[\int_{a}^{x} f(t) e^{i q(t)} d t+c\right]
$$

Received March 24, 1981; revised July 29, 1981.

1980 Mathematics Subject Classification. Primary 65D30; Secondary 65D32. 
so that in general $p$ is as oscillatory as the integrand in (1.1). Therefore, the problem of computing $p$ is no less difficult than the original problem of computing $I$. However, if the functions $f$ and $q^{\prime}$ are slowly oscillatory, then there also exists a slowly oscillatory solution $p_{0}$ of (1.2) (see Appendix). By looking for this $p_{0}$ we avoid all the difficulties caused by the rapid oscillations. Writing the general solution of (1.2) as

$$
p(x)=p_{0}(x)+c e^{-i q(x)}
$$

and using this form in (1.3), we get

$$
I=p_{0}(b) e^{i q(b)}-p_{0}(a) e^{i q(a)} .
$$

Therefore, $p_{0}(a)$ and $p_{0}(b)$ are actually all we need. A problem arises since we do not have any initial condition for $p_{0}$. Furthermore, even if $p_{0}(a)$ were known, any forward integration scheme for (1.2) will develop high oscillations due to round-off errors. However, the rapidly oscillatory homogeneous solution $c e^{-i q(x)}$ can be excluded automatically by solving (1.2) by collocation using slowly oscillatory basis functions. By this method $p_{0}$ is singled out without specifying any boundary conditions. An $n$-point collocation approximation to the solution of (1.2) is defined as

$$
p_{n}(x)=\sum_{k=1}^{n} \alpha_{k} u_{k}(x)
$$

where $\left\{u_{k}\right\}_{k=1}^{n}$ are some linearly independent basis functions, and the coefficients $\left\{\alpha_{k}\right\}_{k=1}^{n}$ are determined by the $n$ collocation conditions

$$
L^{(1)} p_{n}\left(x_{j}\right)=f\left(x_{j}\right), \quad j=1,2, \ldots, n,
$$

where $\left\{x_{j}\right\}_{j=1}^{n}$ are distinct points in $[a, b]$. As is shown in Section 3, it is preferable in this case to choose the endpoints $a$ and $b$ as collocation points. The procedure suggested for an $n$-point approximation $I_{n}$ to $I=\int_{a} b f(t) e^{i q(t)} d t$ is as follows:

(a) Choose a set of linearly independent functions $\left\{u_{k}\right\}_{k=1}^{n}$ (these functions should be suitable for approximating functions with an oscillatory nature similar to that of $f$ ).

(b) Solve, for $\left\{\alpha_{k}\right\}_{k=1}^{n}$, the system of collocation equations

$$
\sum_{k=1}^{n} \alpha_{k} u_{k}^{\prime}\left(x_{j}\right)+i q^{\prime}\left(x_{j}\right) \sum_{k=1}^{n} \alpha_{k} u_{k}\left(x_{j}\right)=f\left(x_{j}\right), \quad j=1,2, \ldots, n,
$$

where $\left\{x_{j}\right\}_{j=1}^{n}$ are regularly distributed in $[a, b], a=x_{1}<x_{2}<\cdots<x_{n}=b$.

(c) Compute the approximation $I_{n}$ to $I$ as

$$
I_{n}=\sum_{k=1}^{n} \alpha_{k} u_{k}(b) e^{i q(b)}-\sum_{k=1}^{n} \alpha_{k} u_{k}(a) e^{i q(a)} .
$$

The above procedure can also be viewed as the expansion method [1, p. 122] using the functions

$$
\phi_{k}(x)=u_{k}^{\prime}(x)+i q^{\prime}(x) u_{k}(x), \quad k=1,2, \ldots, n,
$$

as basis functions for interpolating $f$ at $x_{1}, x_{2}, \ldots, x_{n}$. The "moments" of $\phi_{k}$ required for the application of the expansion method are simply

$$
\psi_{k}=\int_{a}^{b} \phi_{k}(x) e^{i q(x)} d x=u_{k}(b) e^{i q(b)}-u_{k}(a) e^{i q(a)} .
$$


The above differential equation approach provides us with a better recognized tool for investigating this procedure. By analyzing the differential equation one may be able to find a set $\left\{u_{k}\right\}_{k=1}^{n}$ suitable for the collocation approximation (see Example 4). One may also be able to use some results on the choice of collocation points [2] (with the restrictions $x_{1}=a$ and $x_{n}=b$ ).

We remark that a similar idea can be used to evaluate rapidly oscillatory sums of the type

$$
\sum_{m=0}^{N} f(m) e^{i q(m)}
$$

We proceed by presenting the method for the two-dimensional case. The generalization to higher dimensions is evident.

2. The Procedure for Two-Dimensional Integrals Over Rectangular Regions. Here we consider integrals of the form

$$
I=\int_{a}^{b} \int_{c}^{d} f(x, y) e^{i q(x, y)} d x d y,
$$

where $f$ is nonoscillatory, $q_{x}(x, y) \gg(b-a)^{-1}$, and $q_{y}(x, y) \gg(d-c)^{-1}$. The method of Section 1 is easily adaptable to this case, only here we require knowledge of $f, q_{x}, q_{y}$ and $q_{x y}$ over the rectangle $[a, b] \times[c, d]$ and the values of $q$ at its corners.

In analogy to the differential equation (1.2) we introduce here the differential equation

$$
L^{(2)} p=p_{x y}+i q_{y} p_{x}+i q_{x} p_{y}+\left(i q_{x y}-q_{x} q_{y}\right) p=f .
$$

If we could find a solution $p$ of this equation, then the integral $I$ is readily obtained as

$$
I=p(b, d) e^{i q(b, d)}-p(a, d) e^{i q(a, d)}-p(b, c) e^{i q(b, c)}+p(a, c) e^{i q(a, c)} .
$$

This can be shown by using the equality

$$
\frac{\partial^{2}}{\partial x \partial y}\left[p e^{i q}\right]=\left[p_{x y}+i q_{y} p_{x}+i q_{x} p_{y}+\left(i q_{x y}-q_{x} q_{y}\right) p\right] e^{i q}
$$

Here also we are going to use the collocation technique with "nonoscillatory" basis functions to find an approximation to the nonoscillatory solution $p_{0}$ of Eq. (2.2), i.e.,

(a) we choose a set of suitable "nonoscillatory" linearly independent functions $\left\{u_{k}\right\}_{k=1}^{n}$.

(b) We solve, for $\left\{\alpha_{k}\right\}_{k=1}^{n}$, the system of collocation conditions

$$
L^{(2)}\left[\sum_{k=1}^{n} \alpha_{k} u_{k}\right]\left(x_{j}, y_{j}\right)=f\left(x_{j}, y_{j}\right), \quad j=1,2, \ldots, n,
$$

where $\left\{\left(x_{j}, y_{j}\right)\right\}_{j=1}^{n}$ are regularly distributed in $[a, b] \times[c, d]$. Here also it is advisable to include the corners as collocation points.

(c) Form the approximation $I_{n}$ to $I$ by using the approximation $p_{n}=\sum_{k=1}^{n} \alpha_{k} u_{k}$ instead of $p$ in (2.3).

3. Practical Application of the Method and Numerical Experiments. The method described above reduces the problem of integrating rapidly oscillatory functions to solving linear systems ((1.9) or (2.5)). To avoid the difficulties of large linear systems, 
we must keep a low order $n$ of the approximations and improve the accuracy by subdividing the given domain of integration. Another possibility is to use finite support basis functions, e.g., $B$-splines, in the collocation approximation to obtain linear systems with banded (or block banded) matrices. In many cases a clever choice of the basis functions $\left\{u_{k}\right\}$ can yield a very good approximation to the integral over a large or even infinite interval. For example, in the one-dimensional case, if $q(x)$ is a polynomial in $x$ and if $f(x)$ is of the form

$$
f(x)=r(x) e^{s(x)} \text {, }
$$

where $r$ and $s$ are also polynomials in $x$, then by inspection of Eq. (1.2) it is clear that $\left\{u_{k}\right\}$ should be of the form

$$
u_{k}(x)=v_{k}(x) e^{s(x)},
$$

where $v_{k}$ are polynomial (or spline) basis functions.

In the first two examples we consider integrals of the form

$$
\int_{D} f(\mathbf{x}) e^{i W(\mathbf{n} \cdot \mathbf{x}+c \mathbf{x} \cdot \mathbf{x})} d \mathbf{x}
$$

where $W$ is a large parameter and $f$ is of the type (3.1). $D$ is a finite interval in the one-dimensional case $\mathbf{x}=x$ and a rectangle in the two-dimensional case $\mathbf{x}=(x, y)$.

Example 1.

$$
I=\int_{0}^{1} \sin x e^{i W\left(x+c x^{2}\right)} d x
$$

We chose to approximate $p_{0}$ of (1.5) by a polynomial using the monomial basis functions

$$
u_{k}(x)=x^{k-1}, \quad k=1,2, \ldots, n,
$$

and the coefficients $\left\{\alpha_{k}\right\}$ in (1.10) where computed by solving (1.9) with equidistant collocation points $x_{j}=(j-1) /(n-1), j=1, \ldots, n$. An IMSL routine (LEQ2C) was used to solve this complex linear system, and the results were compared to those obtained by Romberg integration (using the routine DCADRE of IMSL).

For the case $W=500$ and $c=1$ it takes about $3 \mathrm{cpu}$ seconds to evaluate $\operatorname{Re} I$ to 5 correct significant figures, $\operatorname{Re} I=4.59859 \ldots \times 10^{-4}$, by DCADRE. The computation of $I_{n}$ takes less than $0.1 \mathrm{cpu}$ seconds for $n \leqslant 10$ giving good approximations to $I$

$$
\operatorname{Re} I_{5}=4.60098 \times 10^{-4}, \quad \operatorname{Re} I_{10}=4.59863 \times 10^{-4} \text {. }
$$

For larger values of $W$ it becomes of course harder for DCADRE to compute $I$. Surprisingly, however, it was found that the approximation $I_{n} \sim I$ improves as $W$ increases, i.e., more significant figures are obtained by $I_{n}$ with fixed $n$. To explain this phenomenon, we use an asymptotic property of oscillatory integrals: If $q^{\prime}$ does not vanish on $[a, b]$, then

$$
\int_{a}^{b} F(x) e^{i W q(x)} d x= \begin{cases}O(1 / W) & \text { as } W \rightarrow \infty \\ O\left(1 / W^{2}\right) & \text { if } F(a)=F(b)=0\end{cases}
$$


Using (3.6), it is clear that the integral (3.4) is of the order $1 / W$ as $W \rightarrow \infty$. On the other hand, the error in $I_{n}$ can be written as

$$
I-I_{n}=\int_{a}^{b}\left(f(x)-L^{(1)} p_{n}(x)\right) e^{i W q(x)} d x .
$$

Using the endpoints $a$ and $b$ as collocation points in (1.9), the integrand in (3.6) vanishes at $a$ and $b$ and, by (3.6),

$$
I-I_{n}=O\left(1 / W^{2}\right) \text { as } W \rightarrow \infty .
$$

Thus, if $f$ does not vanish at the endpoints, the relative error reduces as $W$ increases

$$
\frac{I-I_{n}}{I}=O\left(\frac{1}{W}\right) \text { as } W \rightarrow \infty \text {. }
$$

Example 2.

$$
I(W, c)=\int_{0}^{1} \int_{0}^{1} \cos (x+y) e^{i W\left[x+y+c\left(x^{2}+y^{2}\right)\right]} d x d y .
$$

Here also we used monomials as basis functions

$$
\left\{u_{k}\right\}_{k=1}^{n^{2}}=\left\{x^{i} y^{j} \mid 0 \leqslant i, j \leqslant n-1\right\}
$$

and a square grid of $n^{2}$ collocation points

$$
\left\{\left(\frac{i}{n-1}, \frac{j}{n-1}\right) \mid 0 \leqslant i, j \leqslant n-1\right\} .
$$

We denote the resulting approximation by $I_{n^{2}}(W, c)$; its computation involves solving a complex linear system of order $n^{2}$.

For $W=100$ and $c=1$ we got

$$
I_{9}(100,1)=-8.572 \times 10^{-5}-3.305 \times 10^{-5} i
$$

and

$$
I_{25}(100,1)=-8.595 \times 10^{-5}-3.231 \times 10^{-5} i,
$$

where the exact value is

$$
I(100,1)=-8.597 \times 10^{-5}-3.212 \times 10^{-5} i .
$$

Here it can be shown that

$$
I(W, c)=O\left(1 / W^{2}\right) \quad \text { as } W \rightarrow \infty
$$

and

$$
I(W, c)-I_{n^{2}}(W, c)=O\left(1 / W^{3}\right) \quad \text { as } W \rightarrow \infty .
$$

Therefore, as in the one-dimensional case, the results improve as $W$ increases: For $W=10^{4}$ and $c=1$ we got

$$
I_{25}\left(10^{4}, 1\right)=-6.92088 \times 10^{-9}+2.53659 \times 10^{-9} i
$$

while

$$
I\left(10^{4}, 1\right)=-6.92072 \times 10^{-9}+2.53674 \times 10^{-9} i
$$

Example 3.

$$
I=\int_{0}^{1} \exp \left(\frac{i x}{1-x}\right) d x
$$


In this case the integrand oscillates slowly near $x=0$ and infinitely rapidly as $x$ tends to 1 . It is an example of integrals obtained by transforming integrals oscillating over an infinite range to a finite interval. This particular integral originates from the integral

$$
I=\int_{0}^{\infty} \frac{e^{i x}}{(x+1)^{2}} d x=0.3785504+0.3433779 i
$$

Applying the technique of Section 1 to the integral (3.13), we obtained the differential equation

$$
p^{\prime}+\frac{i}{(1-x)^{2}} p=1
$$

To avoid computational problems at $x=1$, we applied the collocation method (with the monomial basis functions) to the equation

$$
(1-x)^{2} p^{\prime}+i p=(1-x)^{2} \text {. }
$$

The results show that the method can compete with any other method for evaluating the infinite integral (3.14):

$$
\begin{aligned}
I_{5} & =0.3809+0.3442 i \\
I_{10} & =0.378566+0.343380 i \\
I_{15} & =0.3785503+0.3433777 i
\end{aligned}
$$

\section{Example 4.}

$$
I(W)=\int_{0}^{2 \pi} \log x \sin W x d x
$$

This is an example of an oscillatory integral with a singularity, also considered in [1, p. 126]. Writing $I(W)$ as

$$
I(W)=\operatorname{Im}\left\{\int_{0}^{2 \pi} \log x e^{i W x} d x\right\}
$$

we can apply the method of Section 1, i.e., we look for an approximation to the nonoscillatory solution $p_{0}(x)$ of the differential equation

$$
L^{(1)} p(x)=p^{\prime}(x)+i W p(x)=\log x .
$$

It can easily be verified that near $x=0$ the solution of (3.18) can be expanded as

$$
p(x)=\sum_{i=0}^{\infty} a_{i} x^{i}+\log x \sum_{i=0}^{\infty} b_{i} x^{i}
$$

Therefore, we look for an approximation $p_{n}=\sum_{k=1}^{n} \alpha_{k} u_{k}$ to $p_{0}$ using the basis functions

$$
u_{k}(x)=\left\{\begin{array}{ll}
x^{(k-1) / 2}, & k \text { odd, } \\
x^{k / 2} \log x, & k \text { even, }
\end{array} \quad k \geqslant 1 .\right.
$$


To enable collocation of (3.18) at $x=0$, we must take $\alpha_{2}=1$. Then, since $L^{(1)} u_{2}(x)$ $=L^{(1)}(x \log x)=\log x+1+i W x \log x$, the singular term $\log x$ can be subtracted from both sides of the collocation equations (1.8).

To get satisfactory approximations we subdivided the integral as

$$
\int_{0}^{2 \pi}=\int_{0}^{0.2}+\int_{0.2}^{1}+\int_{1}^{2}+\int_{2}^{3}+\int_{3}^{4}+\int_{4}^{5}+\int_{5}^{2 \pi}
$$

The special basis functions in (3.20) were being used only in the interval $[0,0.2]$, while the simple monomial basis functions in (3.5) were being used in the other six subintervals $[0.2,1], \ldots,[5,2 \pi]$. Denoting by $I_{n}(W)$ the sum of the seven $n$th order approximations to the seven integrals on the right-hand side of (3.21), the following approximations to $I(30)=-0.1938773$ were obtained:

$$
I_{6}(30)=-0.1938870, \quad I_{10}(30)=-0.1938763 .
$$

These approximations are significantly better than those given in [1] using the Filon method.

Appendix. We would like to show that if $f$ and $q$ are slowly oscillatory and $\left|q^{\prime}(x)\right| \gg 1$, then the differential equation $((1.2))$

$$
L^{(1)} p=p^{\prime}+i q^{\prime} p=f
$$

has a particular solution $p_{0}$ which is slowly oscillatory in comparison with the rapidly oscillatory homogeneous solution $e^{i q}$.

Instead of counting zeros we shall use a spectral characterization for the oscillatory behavior of functions. Let us write $q$ as $q(x)=W u(x)$, where $0<\left|u^{\prime}(x)\right| \leqslant 1$, and let $x(\zeta)$ be the inverse of $u(x), u(a) \leqslant \xi \leqslant u(b)$. Actually, we should assume that $f(x(\xi)) / q^{\prime}(x(\xi))$ is slowly oscillatory, i.e., that its spectrum is bounded

$$
\frac{f(x(\xi))}{q^{\prime}(x(\xi))}=\int_{-W_{0}}^{W_{0}} G(w) e^{i w \xi} d w, \quad 0<W_{0} \ll W .
$$

Then the slowly oscillatory particular solution of (A.1) is

$$
p_{0}(x)=W \int_{-w_{0}}^{w_{0}} \frac{G(w) e^{i w u(x)}}{i(w+W)} d w .
$$

This can be verified by computing $L^{(1)} p_{0}$ :

$$
\begin{aligned}
L^{(1)} p_{0}(x) & =p_{0}^{\prime}(x)+i q^{\prime}(x) p_{0}(x) \\
& =W \int_{-W_{0}}^{W_{0}} \frac{G(w)}{i(w+W)}\left[i w u^{\prime}(x) e^{i w u(x)}+i W u^{\prime}(x) e^{i w u(x)}\right] d w \\
& =q^{\prime}(x) \int_{-W_{0}}^{W_{0}} G(w) e^{i w u(x)} d w=q^{\prime}(x) \frac{f(x(u(x)))}{q^{\prime}(x(u(x)))}=f(x) .
\end{aligned}
$$

The particular solution (A.3) is slowly oscillatory in comparison with $e^{i q(x)}$ since its spectrum is bounded in $\left[-W_{0}, W_{0}\right]$ and $W_{0} \ll W$. 
1. P. J. Davis \& P. Rabinowitz, Methods of Numerical Integration, Academic Press, New York, 1975.

2. CARL De Boor \& B. SWartz, "Collocation at Gaussian points," SIAM J. Numer. Anal., v. 10, 1973, pp. 582-606.

3. L. N. G. Filon, “On a quadrature formula for trigonometric integrals," Proc. Roy. Soc. Edinburgh, v. 49,1928 , pp. $38-47$.

4. E. A. FlinN, "A modification of Filon's method of numerical integration," J. Assoc. Comput. Mach., v. 7, 1960, pp. 181-184.

5. I. M. Longman, “A method for the numerical evaluation of finite integrals of oscillatory functions," Math. Comp., v. 14, 1960, pp. 53-59. 